\title{
Diagnóstico y tratamiento de los trastornos gastrointestinales del eje cerebro-intestino en niños menores de 2 años
}

\section{Diagnosis and treatment of Gut-Brain Axis Disorders in children younger than 2 years old}

Karen Rubí Ignorosa-Arellano, ${ }^{1}$ Erick Manuel Toro-Monjaraz, ${ }^{1}$ Ericka Montijo-Barrios, ${ }^{2}$ Flora Elva Zárate-Mondragón, ${ }^{2}$ José Francisco Cadena-León, ${ }^{2}$ Roberto Cervantes-Bustamente, ${ }^{2}$ Fernando Michel-Penichet, ${ }^{3}$ María de los Ángeles Estrella González-Camid, ${ }^{3}$ Ulises Leal-Quiroga, ${ }^{3}$ Carlos Iván Oyervides-García, ${ }^{3}$ Carlos Mario Méndez-Nieto, ${ }^{3}$ Marcela Romero-Alcaraz, ${ }^{4}$ Alicia Elizabeth Robledo-Galván, ${ }^{4}$ Karla Alejandra Santos-Jasso, ${ }^{5}$ José Luis Gaitán-Morán, ${ }^{6}$ Alejandro Serrano-Sierra, ${ }^{7}$ Salvador Vázquez-Ortiz, ${ }^{8}$ Román González-Rubio, ${ }^{8}$ Octavio Jesús Mendoza-Gómez, ${ }^{9}$ Jaime Alfonso Ramírez- Mayans ${ }^{10}$

\section{INTRODUCCIÓN}

Los trastornos gastrointestinales funcionales son la principal causa de consulta en el primer año de vida, tanto para el pediatra como para el médico general que atiende niños.

Su naturaleza de origen, sin daño anatómico estructural o bioquímico aparente $^{2} y$, sobre todo, la edad de presentación en menores de un año, específicamente en los primeros seis meses de vida, así como su manifestación clínica, generan gran angustia en los padres y el pediatra. ${ }^{3}$

En algunos de estos niños se practican procedimientos diagnósticos: radiografías de abdomen, serie esófago gastroduodenal, pH-metría, endoscopia y, sobre todo, cambios frecuentes de la fórmula láctea. ${ }^{1,3-5}$

Para evitar complicaciones a largo plazo, el médico debe establecer de manera oportuna un diagnóstico y tratamiento adecuado, y reafirmarle a los padres que con el tratamiento adecuado su hijo tendrá una evolución favorable. ${ }^{6}$

Esto es importante porque a futuro, si estos niños no reciben el tratamiento adecuado, pueden experimentar: trastornos del sueño, de alimentación, de conducta, síndrome de ansiedad, entre otros, consecuencias que implican mayor costo por su atención. ${ }^{4,7}$

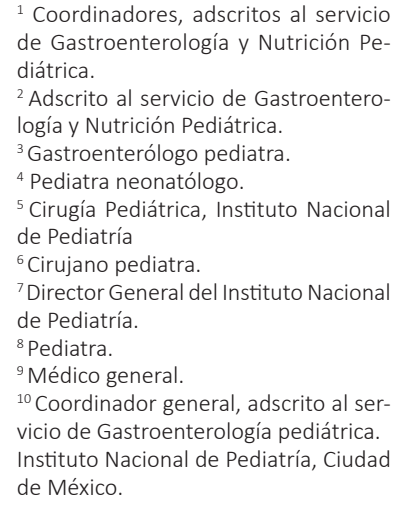

Recibido: 26 de marzo 2018

Aceptado: 21 de septiembre 2018

Correspondencia

Karen Rubí Ignorosa Arellano

karenignorosaa@gmail.com

Este artículo debe citarse como Ignorosa-Arellano KR, et al. Diagnóstico y tratamiento de los trastornos gastrointestinales del eje cerebrointestino en niños menores de 2 años. Acta Pediatr Mex. 2018;39(6):376-384. 


\section{METOdOLOGÍA}

Se presentan, a manera de cuestionario, los trastornos gastrointestinales funcionales más frecuentes en niños menores de dos años, su forma de presentación, señales de alarma, diagnóstico y tratamiento; todo esto con base en los criterios de Roma IV, aplicándolos a la práctica diaria y a la población mexicana.

\section{Trastornos gastrointestinales funcionales en niños menores de 2 años}

\section{¿Cómo se definen los trastornos gastrointestinales funcionales?}

Como inmadurez del tracto gastrointestinal, sin evidencia de alguna afección orgánica o enfermedad subyacente, ni alteraciones bioquímicas. Son inespecíficos, transitorios y no afectan el desarrollo y crecimiento de los niños. ${ }^{2,5,6,8}$

Los niños con trastornos gastrointestinales funcionales son sanos, lo que incluye un buen estado clínico, con adecuado desarrollo antropométrico y neurológico. Si bien son transitorios y benignos, a los padres les genera angustia; por lo tanto, no debe pasar inadvertida la historia psicosocial de los padres y entenderlos para generar empatía. ${ }^{6}$

\section{¿Cuáles son los principales trastornos gastrointestinales funcionales en lactantes?}

Conforme a los criterios de Roma IV son: regurgitación, síndrome de rumiación, síndrome de vómitos cíclicos, cólico, diarrea funcional, disquecia y estreñimiento. ${ }^{3}$ Sin embargo, esta revisión se enfoca en los que son motivo frecuente de consulta en nuestra población: regurgitación, estreñimiento, cólico y disquecia. .,7 $^{2}$
¿Cuál es la prevalencia mundial de los trastornos gastrointestinales funcionales?

En el primer año de vida es de alrededor de 40-60\%; regurgitación en 30\%, cólico en 20\% y estreñimiento en $15 \%$, mientras que la disquecia es de $1-6 \%{ }^{4,7,9}$

En Colombia, Panamá y Nicaragua se llevó a cabo un estudio para conocer la prevalencia de los trastornos gastrointestinales funcionales y los más comunes fueron: cólico (23\%), disquecia $(15 \%)$, estreñimiento $(10 \%)$ y regurgitación $10 \% .{ }^{10}$

\section{¿Cuál es el papel de la microbiota} gastrointestinal en los trastornos gastrointestinales funcionales?

La modificación en la microbiota gastrointestinal; es decir, una disbiosis, puede ser provocada por la vía de nacimiento (cesárea), alimentación con fórmulas, ausencia de lactancia materna, tratamiento con antimicrobianos pre y posnatalmente. Estos cambios pueden favorecer la aparición de algunos de estos trastornos gastrointestinales funcionales. ${ }^{11,12}$ Por esta razón, las fórmulas con pre o probióticos pueden ser una opción terapéutica para favorecer el restablecimiento de la microbiota gastrointestinal en estos niños. ${ }^{13}$

Entre los efectos benéficos asociados con la microbiota en el metabolismo trófico e inmunológico destacan: funciones de barrera en la mucosa y sistémicos, inhibición del crecimiento de bacterias patógenas, estimulación del sistema inmunológico local (tejido linfoide asociado con intestino: GALT) y sistémico, transformación de ácidos biliares, síntesis de ácidos grasos de cadena corta, disminución 
de producción de gas, aumento en el proceso de digestión y absorción de nutrimentos esenciales y síntesis de vitaminas y complejo B. Debido a esto, la microbiota gastrointestinal juega un papel fundamental en el crecimiento, desarrollo de inmunidad y nutrición. ${ }^{11,12,14,15}$

¿Cuál es la relación del eje cerebro-intestino con los trastornos gastrointestinales funcionales?

Estos trastornos emergen de la interacción de múltiples sistemas periféricos (microbioma, inflamación alterada de la mucosa e hipersensibilidad visceral) y el cerebro, que interactúan entre ellos en una forma bidireccional que conduce a la manifestación de los trastornos gastrointestinales funcionales desde etapas tempranas de la vida. ${ }^{16,17}$

\section{¿Cuál es el pronóstico, evolución y consecuencias a largo plazo en los niños con trastornos gastrointestinales funcionales?}

En general, tienen buen pronóstico y remiten alrededor de los seis meses, dependiendo de la etapa de maduración y desarrollo del niño. Sin embargo, de no tener un adecuado diagnóstico y tratamiento pueden tener consecuencias a largo plazo. Entre las principales destacan los trastornos del sueño, de alimentación, de conducta, síndrome de ansiedad, entre otros. ${ }^{7,18-20}$

En niños con cólico o irritabilidad debe descartarse el síndrome del niño sacudido, por lo que la intervención temprana puede, incluso, evitar este tipo de complicaciones. ${ }^{21,22,23}$

\section{Regurgitación}

\section{¿Cuál es la definición de regurgitación?}

Es el paso del contenido gástrico a la faringe o a la boca sin contracción del músculo diafragma. El contenido es expulsado por la boca, sin esfuerzo y en forma no violenta (se dice comúnmente que el niño "devuelve el alimento"). Puede considerarse fisiológica, en un niño sano, si no hay síntomas asociados, ni evidencia de complicaciones. ${ }^{24-27}$

¿Cómo se define el reflujo gastroesofágico?

Es el paso involuntario del contenido gástrico al esófago, manifestado comúnmente como regurgitación o vómito. ${ }^{25}$

¿Qué es la enfermedad por reflujo gastroesofágico?

Es cuando existen complicaciones digestivas y extra-digestivas derivadas del paso del contenido gástrico al esófago y otros órganos. Estas complicaciones pueden ser: esofagitis, sangrado del tubo digestivo alto, neumopatía crónica, estenosis, detención de peso y talla, entre otros. ${ }^{24-27}$

¿Cuál es la prevalencia de regurgitación en lactantes?

La prevalencia mundial se estima en 30\%. ${ }^{4,7}$ En México se desconoce, pero puede considerarse como una manifestación frecuente en los primeros seis meses de vida.

De acuerdo con los criterios de Roma IV, la regurgitación se considera funcional cuando:

- Existen dos o más regurgitaciones al día por más de tres semanas, sin síntomas como: arqueo, hematemesis, aspiración, apnea, falla de crecimiento, alteración de la mecánica de deglución o posturas anormales. Deben concurrir ambos criterios en lactantes por demás sanos de 3 semanas a 12 meses de edad. ${ }^{6}$

¿Qué importancia tiene la técnica de alimentación y su relación con la regurgitación?

El médico debe interrogar acerca de la técnica de alimentación porque la extracción inadecuada 
Ignorosa-Arellano KR, et al. Trastornos gastrointestinales del eje cerebro-intestino

del aire deglutido, la mayor frecuencia de tomas al día sin respetar el tiempo de vaciamiento gástrico, la preparación incorrecta de la fórmula, y el volumen y la osmolaridad pueden ser causa de regurgitación, sin que ésta sea un trastorno gatrointestinal primario..$^{24,25,27}$

\section{¿Cuáles son los datos de alarma en niños con regurgitación?}

Detención del peso y talla, dificultad para deglutir, pérdida de peso, arqueo (posición de Sandifer), vómito biliar, fontanela abombada, tos crónica (neumopatía), sangrado de tubo digestivo, anemia crónica, laringoespasmo, regurgitación que dura más allá de los 12 meses de edad. Cuando se manifieste cualquiera de estos signos y síntomas debe establecerse el diagnóstico diferencial con otros padecimientos..$^{25,28,29}$

\section{¿Cómo se establece el diagnóstico de regurgitación?}

A la fecha, se acepta que el diagnóstico es clínico, requiere una adecuada historia clínica y exploración física y no amerita estudios de gabinete como: serie esófago gastroduodenal, determinación de $\mathrm{pH}$ intraesofágico de 24 horas, impedanciometría, endoscopia, gammagrama de vaciamiento gástrico, entre otros. ${ }^{6,25,26,27,30}$

\section{¿En quiénes están indicados los diferentes} procedimientos diagnósticos?

En los casos en que sea necesario descartar alguna afección orgánica: malformaciones congénitas del tracto gastrointestinal (hernia hiatal, malrotación intestinal, membrana duodenal, entre otros) o bien cuando se manifiestan los signos de alarma señalados. ${ }^{24,25}$

\section{¿Cuál es el tratamiento de regurgitación en un niño alimentado con leche humana?}

Indicar una adecuada técnica de alimentación, en especial acerca de horarios de tomas, fre- cuencia de amamantamiento, de acuerdo con los conocimientos del tiempo de vaciamiento gástrico y, finalmente, la extracción correcta del aire deglutido. En caso de regurgitación, no se recomienda alguna dieta especial en la madre. La técnica de alimentación a libre demanda debe ser dirigida, no cada vez que el niño Ilore. Es importante recordar que la causa del Ilanto no necesariamente implica hambre, también puede explicarse por otros factores. ${ }^{25,27,28,29}$

\section{¿Cuál es el tratamiento para la regurgitación en un lactante alimentado con fórmula láctea?}

Al igual que el niño alimentado con leche humana es importante establecer una adecuada técnica de alimentación; es decir, preparación correcta de la fórmula en las diluciones recomendadas, sin adición de endulzantes (miel, aceite, atoles u otros componentes). La frecuencia adecuada, de acuerdo con la edad del niño menor de 6 meses, en general, es cada 3 a 4 horas. ${ }^{25,27,28,29}$

\section{En niños con regurgitación ¿deben indicarse medicamentos?}

En el caso de regurgitación funcional no se recomienda la prescripción de medicamentos. ${ }^{6}$

\section{Estreñimiento}

\section{¿Cómo se define en los lactantes?}

En niños menores de dos años no existe una definición clara de estreñimiento; sin embargo, se considera la ausencia o disminución de evacuaciones menor a 3 por semana, y cuando éstas se logran puede existir dolor, según la consistencia de las heces que pueden ser duras o pastosas. No debe haber datos de alarma que orienten a un daño orgánico (sangrado, detención de peso y talla, entre otros). ${ }^{31,32,33}$ 
Criterios de Roma IV (estreñimiento) para niños menores de 4 años

En niños menores de 4 años deben manifestarse durante un mes de evolución o, al menos, dos de los siguientes: ${ }^{6}$

- Dos o menos evacuaciones por semana

- Antecedente de retención excesiva de heces

- Antecedente de movimientos intestinales dolorosos o duros

- Antecedente de heces de gran diámetro

- Masa fecal grande en el recto

¿Cuál es la prevalencia de estreñimiento en lactantes?

Se desconoce la prevalencia mundial de estreñimiento en niños menores de un año; sin embargo, se estima que es aproximadamente de $15 \%$, dependiendo del tipo de alimentación del niño. ${ }^{7}$ En México se desconoce la verdadera prevalencia.

¿Cuáles son las principales causas de estreñimiento en lactantes?

En lactantes menores de 6 meses la causa es, fundamentalmente, de tipo funcional, quizá relacionada con inmadurez del tracto gastrointestinal. Existen otras causas que deben considerarse, como el cambio de leche humana a fórmula. Otro periodo crítico es el inicio de la alimentación complementaria y la falta de ingesta de líquidos, sobre todo en lugares con temperaturas elevadas. $28,31,32,34$

\section{¿Qué es la disquecia?}

Es la defecación difícil o dolorosa, según los criterios de Roma IV la padecen niños menores de nueve meses y debe incluir: pujo, Ilanto, durante 10 a 20 minutos, antes del paso de evacuaciones blandas, sin evidencia de algún problema orgánico.
Se cree se debe a falta de coordinación entre la presión intra abdominal y la relajación del piso pélvico. Suele resolverse al paso de los días. ${ }^{6}$

¿Qué importancia tiene la técnica de alimentación como causa de estreñimiento en lactantes?

La técnica de alimentación es un factor que puede modificar el patrón de evacuaciones en los lactantes, desde la frecuencia de las tomas, volumen, preparación inadecuada de la fórmula, entre otros. Hasta ahora se carece de publicaciones científicas que demuestren que la técnica de alimentación sea un factor preponderante como causa de estreñimiento funcional en niños menores de un año. $28,29,31,34$

¿Qué relación tiene la alimentación complementaria en niños con estreñimiento?

Con la introducción de alimentos sólidos en la dieta disminuye la ingesta de líquidos, además de la restricción del consumo de fibra contenida en las cáscaras de frutas y algunos vegetales, y el consumo de cereales precocidos, que pueden favorecer el estreñimiento. ${ }^{29,31}$

¿Cuáles son los datos de alarma en un niño con estreñimiento?

Detención del crecimiento, sangrado gastrointestinal, vómitos, ausencia de evacuaciones (meconio) en las primeras 48 horas de vida, antecedente de enterocolitis necrotizante, ámpula rectal vacía al tacto, distensión abdominal importante, retraso psicomotor, malformaciones recto anales, manifestaciones de otras enfermedades (hipotiroidismo), hipotonía. ${ }^{28,29,34}$

$¿$ ¿Debe realizarse tacto rectal en todos los lactantes con estreñimiento?

Cada paciente es distinto, solo debe realizarse en casos donde haya datos de alarma (ya mencionados). ${ }^{33,34}$ 
Ignorosa-Arellano KR, et al. Trastornos gastrointestinales del eje cerebro-intestino

¿Está indicada alguna prueba diagnóstica en lactantes con estreñimiento?

En estreñimiento funcional no es necesario realizar estudios complementarios, a menos que existan datos de alarma. ${ }^{6}$

\section{¿Cuál es el tratamiento en un niño con} estreñimiento, alimentado exclusivamente con leche humana?

Debe recordarse que el estreñimiento son evacuaciones duras, si bien es poco frecuente que un niño alimentado con leche humana lo padezca debe aumentarse la ingesta de líquidos en la madre, corregir la técnica de alimentación y, en caso de persistir, puede utilizarse como auxiliar leche de magnesia o lactulosa. ${ }^{28,29}$

En caso de no poder suministrar leche humana, ¿qué fórmula láctea está indicada?

Debe considerarse una fórmula con nutrimentos que puedan auxiliar en el tratamiento del estreñimiento: algarrobo, beta palmitato, simbióticos, alfa lactoalbúmina, relación calcio-fósforo, y cantidad de lactosa. ${ }^{34-37}$

En niños mayores de 6 meses ¿qué tipo y cantidad de fibra está recomendada?

Entre los 6 y 12 meses deben introducirse, de manera gradual, cereales, verduras y leguminosas, aumentando la cantidad de fibra en la dieta a 5 g/día antes del primer año de vida. La Academia Americana de Pediatría recomienda $0.5 \mathrm{~g} / \mathrm{kg}$ de fibra al día, hasta 35 g como cantidad máxima. Otra recomendación del consumo diario de fibra para niños mayores de 2 años es la edad (años) + 5 a 10 g al día. ${ }^{13,38,39}$

Si bien aún hay datos insuficientes acerca de la repercusión de la fibra de alimentos completos y cantidad de agua en el tratamiento del estre- ñimiento, se recomienda indicar que el aporte de fibra en la dieta y agua sea en cantidades suficientes, acordes con la edad y sexo. ${ }^{34}$

¿Qué importancia tienen los prebióticos, probióticos y simbióticos en la composición de una fórmula para niños con estreñimiento?

Se carece de suficiente evidencia como para recomendar prebióticos y probióticos como tratamiento de estreñimiento. Existen diversas fórmulas adicionadas con prebióticos, probióticos, simbióticos. ${ }^{39,40}$

\section{¿Está indicado el tratamiento farmacológico en lactantes con estreñimiento?}

En niños menores de seis meses puede indicarse lactulosa (1-3 mL/kg/día) y, en mayores, polietilenglicol $(0.5-1 \mathrm{~g} / \mathrm{kg} / \mathrm{día})$ o leche de magnesia (1-3 mL/kg/día). La duración del tratamiento será el tiempo necesario hasta corregir el problema; es decir, iniciar con dosis-respuesta y mensualmente disminuir el medicamento hasta retirarlo y lograr evacuaciones diarias, blandas y sin dolor. 28,29,34,41

\section{Cólico}

\section{¿Cómo se define el cólico en niños?}

De acuerdo con Wessel, el cólico aparece con episodios de llanto intenso y vigoroso al menos 3 horas en el día, 3 días a la semana durante al menos 3 semanas en un niño aparentemente sano, sin evidencia de daño orgánico-estructural y bien alimentado. ${ }^{42}$

De acuerdo con los criterios de Roma IV, se refiere a un lactante menor de 5 meses de edad al inicio y término de síntomas, con: ${ }^{6}$

- Periodos prolongados y recurrentes de Ilanto, incomodidad, irritabilidad (Ilanto, incomodidad, irritabilidad durante más 
de 3 horas al día, durante 3 o más días a la semana) reportados por los cuidadores, que ocurre sin causa aparente y no puede prevenirse ni resolverse por los cuidadores.

- Sin evidencia de falla en el crecimiento, fiebre o enfermedad.

¿Cuál es la prevalencia del cólico en lactantes?

La prevalencia mundial del cólico en niños es, aproximadamente, de $20 \%$. Su máxima incidencia es a las seis semanas de vida, y es más frecuente en el primer hijo, en hijos de padres fumadores. ${ }^{7,9}$

¿Cuál es el papel de la técnica de alimentación y su relación con el cólico en lactantes?

Una técnica adecuada de alimentación es decisiva para evitar que el niño tenga cólico, por eso debe indicarla personal de salud capacitado. Debe insistirse en la correcta extracción del aire deglutido, respetar los horarios de alimentación, en el caso de ser alimentados con fórmula, no añadir ningún complemento, y su adecuada preparación. $^{28,29,43}$

¿Cuáles son los datos de alarma en un niño con cólico?

Cuando las medidas de intervención no funcionan, hay detención de peso y talla, fiebre intermitente, infecciones de repetición, irritabilidad excesiva, dolor abdominal, signos de atopia, heces con sangre, ansiedad de los padres..$^{28,29,44}$

¿Están indicados los estudios de gabinete para poder establecer el diagnóstico de cólico?

No, pero si existen datos de alarma deben practicarse estudios de acuerdo con la sospecha diagnóstica. ${ }^{6}$
¿Cuál es el diagnóstico diferencial en un niño con cólico?

Cualquier padecimiento que cause Ilanto inexplicable que pueda confundirse con cólico del lactante. Uno de los principales diagnósticos diferenciales es con alergia a la proteína de la leche de vaca, otros de tipo infeccioso son: otitis media, encefalitis, infección de vías urinarias; maltrato, fracturas, invaginación intestinal, entre otros..$^{28,29}$

¿Cuál es el tratamiento en un niño con cólico alimentado con leche humana?

Recomendaciones de puericultura, diferenciar las principales causas del Ilanto, y no suspender la lactancia materna. ${ }^{43}$ Existen otras opciones, como los probióticos, que no se recomiendan para consumo rutinario. ${ }^{45,46,47}$

¿Cuál es el tratamiento en un niño con cólico alimentado con fórmula láctea?

Se carece de consenso acerca del tipo de fórmula que debe indicarse para disminuir los síntomas; sin embargo, existen ciertos nutrimentos contenidos en las fórmulas que pueden disminuir el cólico. En general, debe revisarse la preparación de la fórmula, corregir la técnica de alimentación, evaluar el patrón de evacuaciones y hacer un adecuado interrogatorio clínico y exploración física. ${ }^{28,29}$

¿Qué utilidad tienen los prebióticos y probióticos en las fórmulas lácteas en el tratamiento de un niño con cólico?

En niños alimentados solo con leche humana los probióticos (Lactobacillus reuteri DSM 17938, nivel de evidencia B) han demostrado, en algunos estudios, reducción del llanto y de su duración. ${ }^{46,47}$ Existen diferentes prebióticos adicionados a las fórmulas lácteas; no se recomienda su consumo rutinario. ${ }^{13,39}$ 
Ignorosa-Arellano KR, et al. Trastornos gastrointestinales del eje cerebro-intestino

¿Está indicado el tratamiento farmacológico en niños con cólico?

Se indican: antiespasmódicos, analgésicos, inhibidores de bomba, probióticos, medicina herbolaria, entre otros, pero sin evidencia de que realmente disminuyan los síntomas, ni de que puedan cosumirse rutinariamente. ${ }^{41,43,44}$

\section{CONCLUSIONES}

Los trastornos gastrointestinales funcionales en niños representan un problema frecuente, motivo de consulta habitual al pediatra y generadores de repercusiones que afectan la dinámica familiar. Por esto, el papel del pediatra o médico de primer contacto es muy importante en la atención no solo del niño, sino también de la familia porque su misión es reforzar la ausencia de organicidad, explicar que son trastornos benignos y transitorios debidos a inmadurez del tracto gastrointestinal. El diagnóstico y el tratamiento deben establecerse oportunamente y detectar los signos de alarma y evitar las complicaciones futuras. La puericultura y un adecuado conocimiento de la nutrición infantil son las bases para el tratamiento de estos trastornos funcionales.

\section{REFERENCIAS}

1. Rouster AS, et al. Functional gastrointestinal disorders dominate pediatric gastroenterology outpatient practice. J Pediatr Gastroenterol Nutr. 2016;62(6):847-51. doi: 10.1097/ MPG.0000000000001023

2. Ferreira-Maia AP, et al. Epidemiology of functional gastrointestinal disorders in infants and toddlers: A systematic review. World J Gastroenterol. 2016;22(28):6547. 10.3748/ wjg.v22.i28.6547

3. Pina DI, et al. Prevalence and dietetic management of mild gastrointestinal disorders in milk-fed infants. World J Gastroenterol. 2008;14(2):248-54. doi: 10.3748/wjg.14.248

4. Glanville J, et al. Costs associated with functional gastrointestinal disorders and related signs and symptoms in infants: A systematic review protocol. BMJ Open. 2016;6(8):e0114475. http://dx.doi.org/10.1136/bmjopen-2016-011475

5. Scarpato $E$, et al. Functional Gastrointestinal Disorders in Children: A Survey on Clinical Approach in the Mediterra- nean Area. J Pediatr Gastroenterol Nutr. 2017;64(6):e1426. doi: 10.1097/MPG.0000000000001550

6. Benninga MA, et al. Childhood functional gastrointestinal disorders: Neonate/toddler. Gastroenterology. 2016;150(6):1443-1455e2. https://doi.org/10.1053/j. gastro.2016.02.016

7. Vandenplas $Y$, et al. Prevalence and health outcomes of functional gastrointestinal symptoms in infants from Birth to 12 Months of Age. J Pediatr Gastroenterol Nutr. 2015;61(5):531-7. doi:10.1097/MPG.0000000000000949]

8. Koppen IJN, et al. Motility disorders in infants. Early Hum Dev. 2017;114:1-6. https://doi.org/10.1016/j.earlhumdev.2017.09.005

9. Miranda AL, et al. Prevalence of functional gastrointestinal disorders in infants and toddlers. J Pediatr. 2015;166(3):6849. https://doi.org/10.1016/j.jpeds.2014.11.039

10. Chogle A, et al. Multicountry cross-sectional study found that functional gastrointestinal disorders such as colic and functional dyschezia were common in South American infants. Acta Paediatr. 2018 Jan 4;107(4):708-13. https:// doi.org/10.1111/apa.14196

11. Abrahamsson TR, et al. Microbiota in functional gastrointestinal disorders in infancy: implications for management. Nestle Nutr Inst Workshop Ser. 2017;88:107-15. https:// doi.org/10.1159/000455219

12. Videhult FK, et al. Nutrition, gut microbiota and child health outcomes. Curr Opin Clin Nutr Metab Care. 2016;19(3):208-13. doi: 10.1097/MCO.0000000000000266

13. Horvath A, et al. Probiotics, prebiotics, and dietary fiber in the management of functional gastrointestinal disorders. World Rev Nutr Diet. 2013;108:40-8. https://doi. org/10.1159/000351483

14. Castanys-Muñoz E, et al. Building a beneficial microbiome from birth. Advances in Nutrition 2016;7(1):323-30. https://doi.org/10.3945/an.115.010694

15. Wopereis $\mathrm{H}$, et al The first thousand days - intestinal microbiology of early life: Establishing a symbiosis. Pediatr Allergy Immunol. 2014;25(5):428-38. https://doi. org/10.1111/pai.12232

16. Jones MP, et al. Brain-gut connections in functional GI disorders: Anatomic and physiologic relationships. Neurogastroenterol Motil. 2006;18(2):91-103. https://doi. org/10.1111/j.1365-2982.2005.00730.x

17. Pärtty A, et al. Infant colic is still a mysterious disorder of the microbiota-gut-brain axis. Acta Paediatr Int J Paediatr. 2017;106(4):528-9. https://doi.org/10.1111/apa.13754

18. Hemmi MH, et al. Associations between problems with crying, sleeping and/or feeding in infancy and long-term behavioural outcomes in childhood: A meta-analysis. Arch Dis Child. 2011;96(7):622-9. http://dx.doi.org/10.1136/ adc.2010.191312

19. Rao MR, et al. Long term cognitive development in children with prolonged crying. Arch Dis Child. 2004;89(11):989-92. http://dx.doi.org/10.1136/adc.2003.039198 
20. Savino F, et al. A prospective 10 -year study on children who had severe infantile colic. Acta Paediatr. 2005;94(0):12932. https://doi.org/10.1111/j.1651-2227.2005.tb02169.x

21. Gupta SK. Is colic a gastrointestinal disorder? Curr Opin Pediatr. 2002;14(5):588-92.

22. Bailey SD, et al. Information on Infantile Colic on the World WideWeb. J Pediatr Heal Care. 2013;27(6):443-50. https:// doi.org/10.1016/j.pedhc.2012.04.009

23. Camilleri $\mathrm{M}$, et al. Exploring hypotheses and rationale for causes of infantile colic. Neurogastroenterol Motil. 2017;29(2):1-11. https://doi.org/10.1111/nmo.12943

24. Vandenplas $Y$, et al. Pediatric gastroesophageal reflux clinical practice guidelines: joint recommendations of the North American Society for Pediatric Gastroenterology, Hepatology, and Nutrition (NASPGHAN) and the European Society for Pediatric Gastroenterology, Hepatology, a. J Pediatr Gastroenterol Nutr. 2009;49(4):498-547. doi: 10.1097/MPG.0b013e3181b7f563

25. Ramírez Mayans JA, et al. Consenso médico quirúrgico para el manejo de niños con reflujo gastroesofágico. Acapulco, México 2002. Rev Gastroenterol Mex. 2003;68(3):223-34.

26. Mousa H, et al. Gastroesophageal reflux disease. Pediatr Clin North Am. 2017;64(3):487-505. DOI: https://doi. org/10.1016/j.pcl.2017.01.003

27. Lightdale JR, Gremse DA. Gastroesophageal Reflux: Management Guidance for the Pediatrician. Pediatrics. 2013;131(5):e1684-95.

28. Vandenplas $Y$, et al. Algorithms for managing infant constipation, colic, regurgitation and cow's milk allergy in formula-fed infants. Acta Paediatr Int J Paediatr. 2015;104(5):449-57. https://doi.org/10.1111/apa.12962

29. Vandenplas $Y$, et al. Practical algorithms for managing common gastrointestinal symptoms in infants. Nutrition. 2013;29(1):184-94. https://doi.org/10.1016/j. nut.2012.08.008

30. Czinn SJ, et al. Gastroesophageal reflux disease in neonates and infants.Pediatr Drugs. 2013;15(1):19-27. https://doi. org/10.1007/s40272-012-0004-2

31. Kuizenga-Wessel $\mathrm{S}$, et al. Reporting outcome measures of functional constipation in children from 0 to 4 years of age. J Pediatr Gastroenterol Nutr. 2015;60(4):446-56. doi: 10.1097/MPG.0000000000000631

32. Mugie SM, Di Lorenzo C, Benninga MA. Constipation in childhood. Nat Rev Gastroenterol Hepatol. 2011;8(9):502-11.

33. Tabbers MM, et al. Evaluation and treatment of functional constipation in infants and children: Evidencebased recommendations from ESPGHAN and NASPGHAN. J Pediatr Gastroenterol Nutr. 2014;58(2):258-74. doi: 10.1097/MPG.0000000000000266
34. Remes-Troche JM, et al. Guías de diagnóstico y tratamiento del estreñimiento en México. D) Evaluación y tratamiento del estreñimiento en población pediátrica. Rev Gastroenterol México. 2011;76(2):155-68.

35. Civardi E, et al. Safety, growth, and support to healthy gut microbiota by an infant formula enriched with functional compounds. Clin Nutr. 2017;36(1):238-45. doi: 10.1016/j. clnu.2015.11.006.

36. Nowacki J, et al. Stool fatty acid soaps, stool consistency and gastrointestinal tolerance in term infants fed infant formulas containing high sn-2 palmitate with or without oligofructose: A double-blind, randomized clinical trial. Nutr J. 2014;13(1):105. doi: 10.1186/1475-2891-13-105.

37. Litmanovitz I, et al. Reduced crying in term infants fed high beta-palmitate formula: A double-blind randomized clinical trial. BMC Pediatr. 2014;14(1):1-6. doi: 10.1186/14712431-14-152

38. Koback G. Pediatric Nutrition Handbook. Archives of Pediatrics \& Adolescent Medicine. 1999;153:1205.

39. Wegh CAM, Schoterman MHC, Vaughan EE, Belzer C, Benninga MA. The effect of fiber and prebiotics on children's gastrointestinal disorders and microbiome. $V$ Expert Review of Gastroenterology and Hepatology. Taylor \& Francis, 2017;11:1031-45

40. Wojtyniak K, Szajewska H. Systematic review: probiotics for functional constipation in children. Eur J Pediatr. 2017;176(9):1155-62.

41. Salvatore S, Barberi S, Borrelli O, Castellazzi A, Di Mauro D, Di Mauro G, et al. Pharmacological interventions on early functional gastrointestinal disorders. Ital J Pediatr. 2016;42(1):1-8.

42. Wessel MA, et al. Paroxysmal fussing in infancy, sometimes called colic. Pediatrics. 1954;14(5):421-35.

43. Harb T, et al. Infant colic - What works: A systematic review of interventions for breast-fed infants. J Pediatr Gastroenterol Nutr. 2016;62(5):668-86. doi: 10.1097/ MPG.0000000000001075.

44. 44. Gelfand AA. Infant Colic. Semin Pediatr Neurol. 2016;23(1):79-82. doi: 10.1016/j.spen.2015.08.003.

45. Schreck BA, et al. Probiotics for the Treatment of Infantile Colic: A Systematic Review. J Pharm Pract. 2016:1-9. https://doi.org/10.1177/0897190016634516

46. Savino F, et al. Pediatrics: Lactobacillus reuteri DSM 17938 in Infantile Colic: A Randomized, Double-Blind, PlaceboControlled Trial. Pediatrics. 2010;126(3):e526-33.

47. Szajewska $\mathrm{H}$, et al. Lactobacillus reuteri DSM 17938 for the management of infantile colic in breastfed infants: A randomized, double-blind, placebo-controlled trial. J Pediatr. 2013;162(2):257-62. https://doi.org/10.1016/j. jpeds.2012.08.004 